\title{
Interference with sphingosine 1-phosphate synthesis affects Merkel cell carcinoma proliferation in vitro
}

\section{${ }^{1}$ Bhat Kumble V, ${ }^{1}$ Bernhart E, ${ }^{1}$ Plastira I, ${ }^{2,4}$ Fan K, ${ }^{1}$ Rechberger G, ${ }^{3}$ Wadsack C, ${ }^{4}$ Becker JC, ${ }^{1}$ Sattler W}

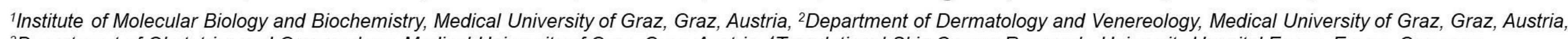

${ }^{3}$ Department of Obstetrics and Gynaecology, Medical University of Graz, Graz, Austria; ${ }^{4}$ Translational Skin Cancer Research, University Hospital Essen, Essen, Germany

\section{Background}

\section{Aims}

Merkel cell carcinoma (MCC) is an aggressive metastatic neuroendocrine skin cancer affecting primarily the elderly population. Age $>50$ years, immune deficiency, Merkel cell polyoma virus (MCPyV) infection, or UV exposure are known risk factors of MCC pathogenesis. The incidence is 0.6 per 100,000 persons and rising all over the world (approximately 1600 new cases have been reported in the USA every year) There is no evidence based effective chemotherapy since the cellular and molecular mechanisms dictating MCC survival and metastasis have remained unclear so far.

Sphingosine-1-phosphate $(\mathrm{S} 1 \mathrm{P})$ is an important bioactive sphingolipid playing a decisive role in intracellular and extracellular signalling pathways, that can promote tumor cell survival, proliferation, migration, and angiogenesis. S1P transmits signals via five different cognate $\mathrm{G}$ protein-coupled receptors termed S1PR1-5. Endogenous S1P levels are regulated by sphingosine kinases (SPHK1 and -2) which are upregulated in many tumors. The objective of this study was to elucidate whether pharmacological interference with S1P synthesis would impact MCC cell proliferation, survival, and apoptosis.

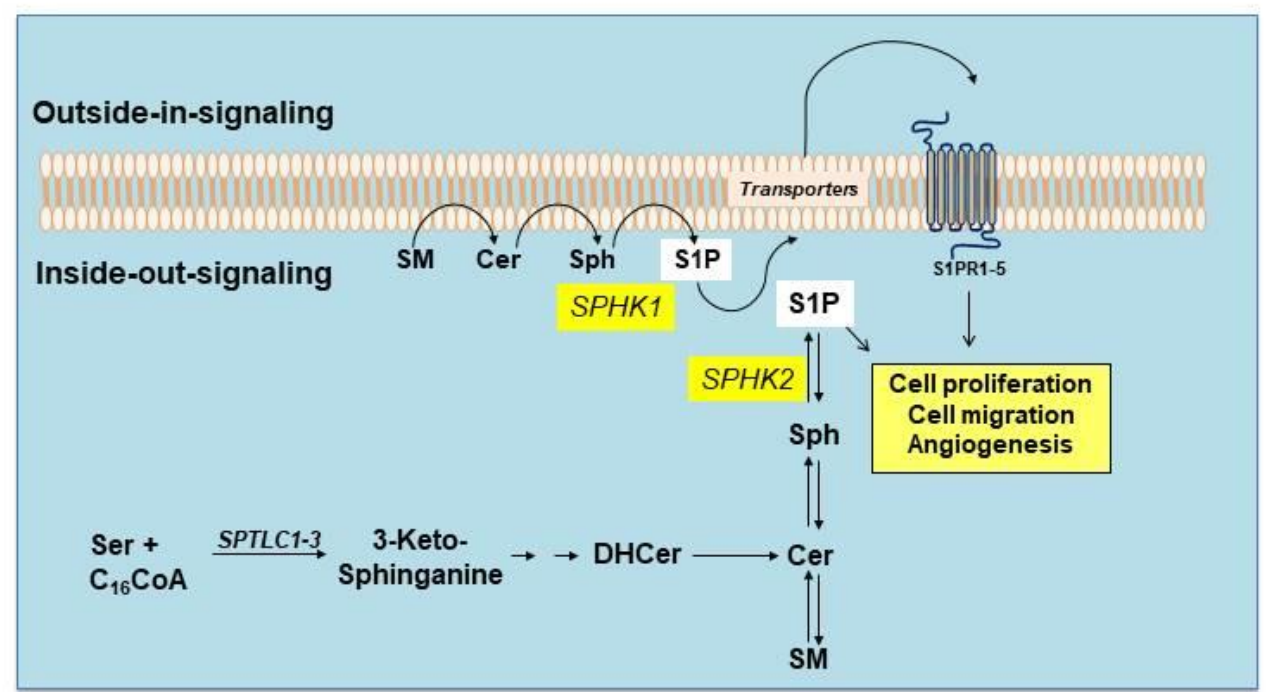

* To characterize expression of sphingosine kinase 1 and 2 (SPHK 1, -2) in MCC cell lines

* To study the outcome of pharmacological inhibition of SPHK1 and 2 by SKI-II on MCC cell proliferation and survival

* To assess the effect of SPHK1 and 2 inhibitor SKI-II on cell viability of nontransformed normal dermal fibroblasts (NHDF)

* To elucidate the effect of SPHK 1 and 2 inhibitor on intracellular S1P, ceramide and sphingomyelin synthesis

* To analyze the mechanism of cell death upon inhibitor treatment

\section{Results}

Characterization of SPHK1 and 2 expression in MCPyV-positive and negative MCC cell lines

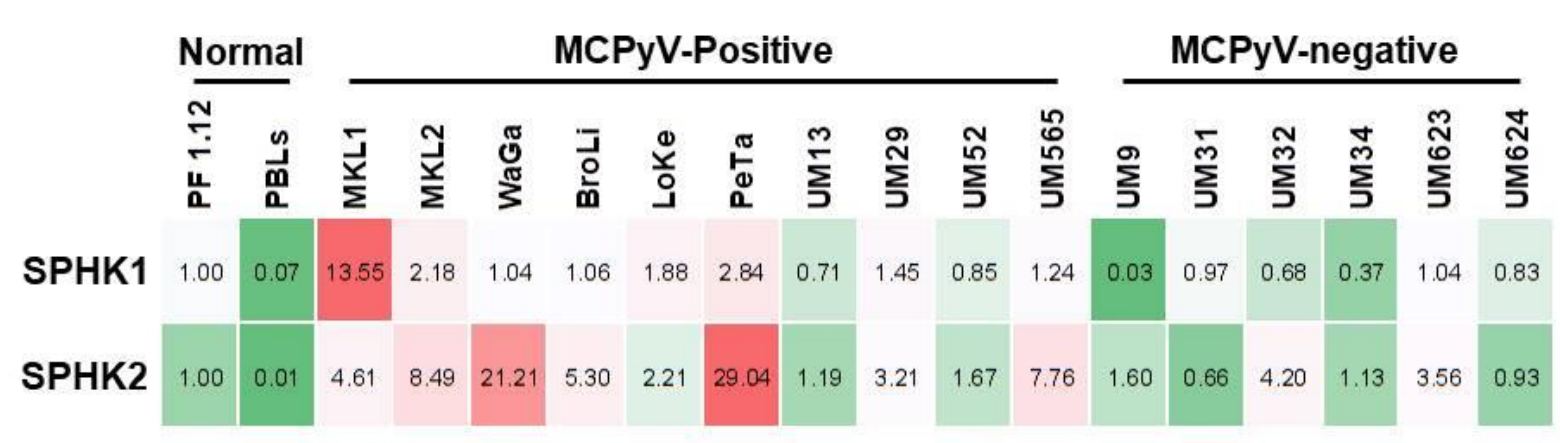

Figure 1: Characterization of SPHK1 and 2 mRNA expressions in MCC cell lines by qRT-PCR. The RNA was isolated and CDNA was prepared from 2 normal cells and 16 MCC cell lines. qRT-PCR was done using primers specific to SPHK1, -2 and RPLPO. The heat map was plotted relative to primary fibroblast (PF 1.12) using $2^{-\triangle \triangle C T}$ method after normalizing the Ct-value to RPLPO.

\section{Pharmacological inhibition of SPHK1 and 2 by SKI-II attenuates MCC cell proliferation}
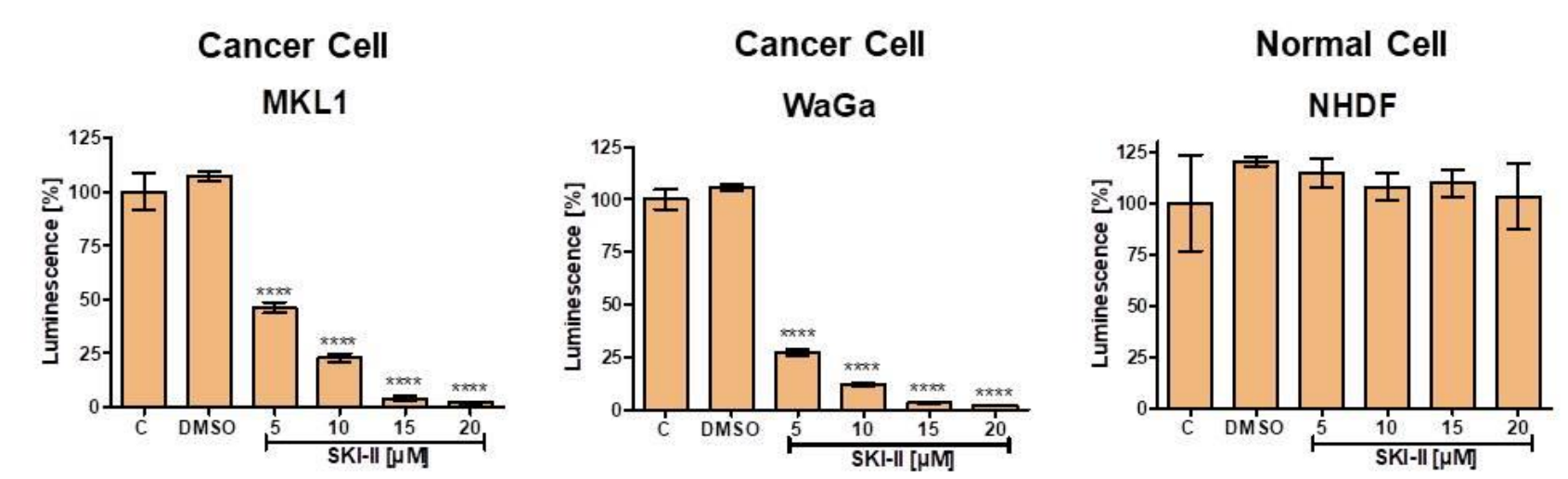

Figure 2 : SKI-II an inhibitor of SPHK 1 and 2 decreases the proliferation of MKL1 and WaGa cells with no effect on NHDF cells. 20,000 cells in $0.5 \%$ FBS media were seeded per well in 96 well plate and treated with DMSO or SKI-II inhibitor. The proliferation of MKL1, WaGa MCC cells and NHDF cells (negative control) were measured after $48 \mathrm{~h}$ of treatment using CellTiter-Glo 3D Cell Viability assay (Promega).

\section{Conclusions}

Data presented here demonstrate that all MCC cell lines tested express SPHK 1 and 2. Intracellular S1P is an important factor regulating tumor cell survival, proliferation and metastasis. Inhibition of intracellular S1P synthesis by inhibiting SPHK function significantly affected MCC cell survival and proliferation in vitro. Pharmacological antagonism of SPHK function in MKL1 and WaGa cells significantly decreased PAKT and intracellular S1P, sphingomyelin level and increased ceramide, caspase 3 and PARP leading to apoptosis of MCC cells. Interestingly, SPHK inhibition was without effect on non-transformed NHDF cells. Our data point towards the possibility that sphingolipid synthesis in MCC could be exploited to interfere with the highly neoplastic behavior of this tumor type.

\section{Acknowledgement}

This study was funded by the Austrian Science Fund (FWF) and the Medical University of Graz (DKMOLIN, Project W1241)

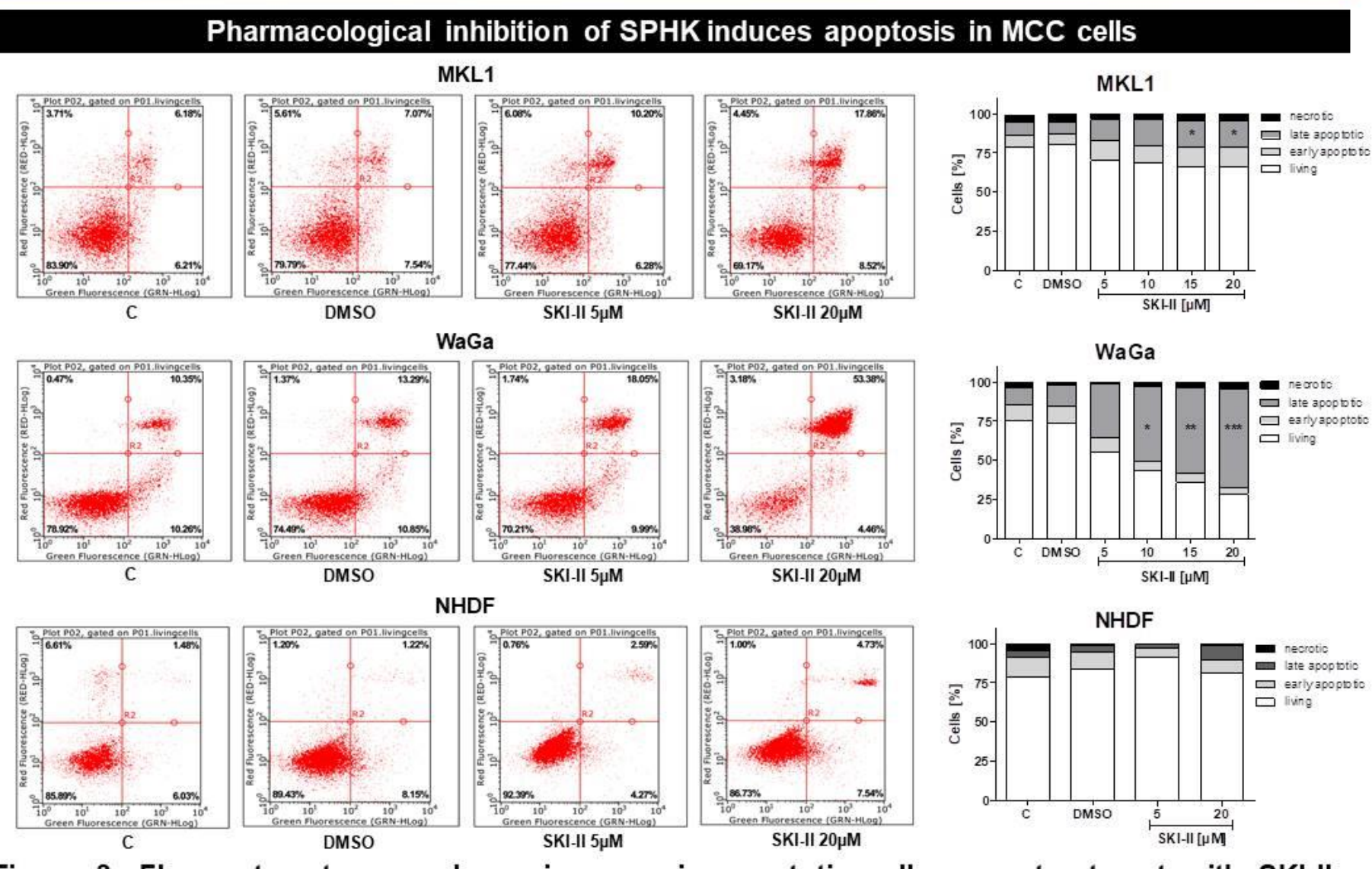

Figure 3: Flow cytometry reveals an increase in apoptotic cells upon treatment with SKI-II. Approximately 750,000 cells were seeded per well and treatment was induced as described above. Cells were harvested after $48 \mathrm{~h}$ of treatment and FITC Annexin V apoptosis detection kit was used to stain and analyse the type of cell death by flow cytometry.

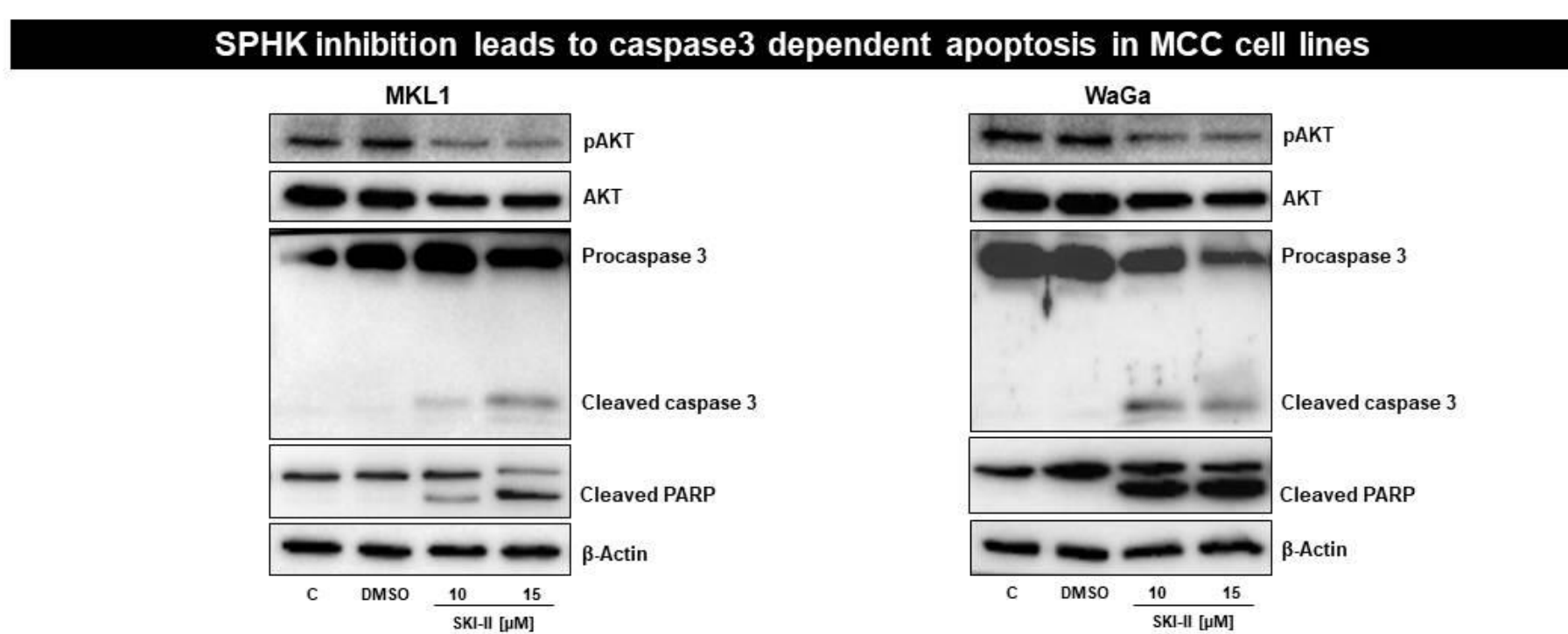

Figure 4: Western blot analysis shows decrease in pAKT level and increase in cleaved caspase 3 and PARP. MKL1 and WaGa cells were incubated with SKI-II inhibitor at the concentrations indicated. Cells were harvested for immuno blotting $48 \mathrm{~h}$ post treatment.
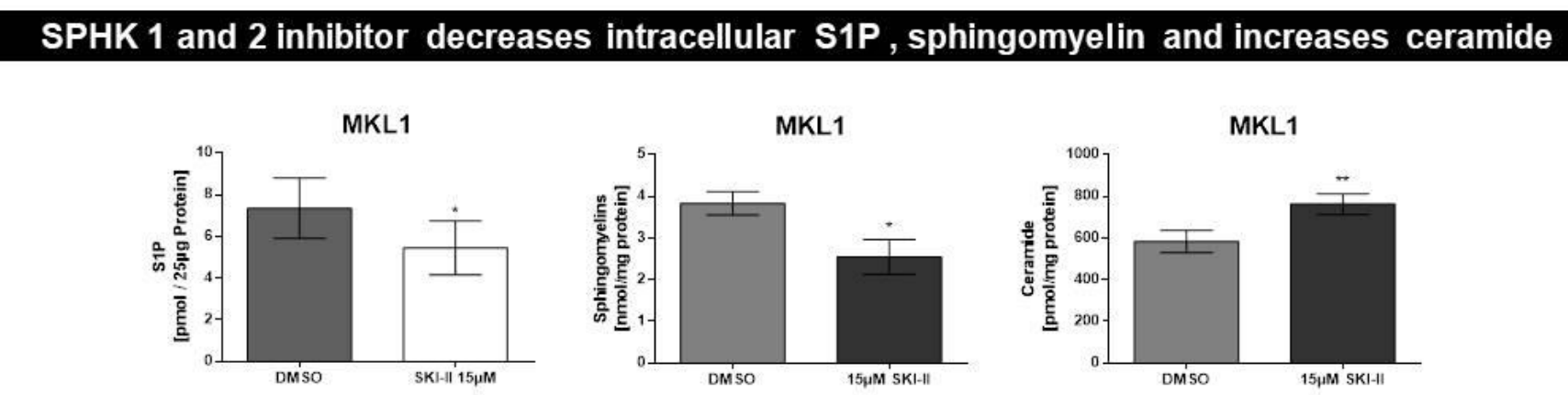

Figure 5: Reduction in intracellular S1P, sphingomyelin and increase in ceramide concentration upon treatment with SKI-II . Intracellular ceramide and sphingomyelin concentration was quantitated by LC-MS and S1P concentration was measured using S1P-ELISA. 